\title{
Strong Collectionwise Normality and M. E. Rudin's Dowker Space
}

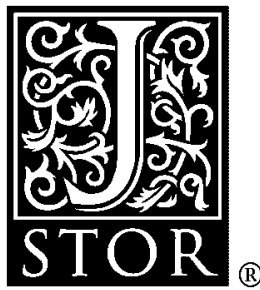

\author{
K. P. Hart
}

Proceedings of the American Mathematical Society, Vol. 83, No. 4. (Dec., 1981), pp. 802-806.

Stable URL:

http://links.jstor.org/sici?sici=0002-9939\%28198112\%2983\%3A4\%3C802\%3ASCNAME\%3E2.0.CO\%3B2-Y

Proceedings of the American Mathematical Society is currently published by American Mathematical Society.

Your use of the JSTOR archive indicates your acceptance of JSTOR's Terms and Conditions of Use, available at

http://www.jstor.org/about/terms.html. JSTOR's Terms and Conditions of Use provides, in part, that unless you have obtained prior permission, you may not download an entire issue of a journal or multiple copies of articles, and you may use content in the JSTOR archive only for your personal, non-commercial use.

Please contact the publisher regarding any further use of this work. Publisher contact information may be obtained at http://www.jstor.org/journals/ams.html.

Each copy of any part of a JSTOR transmission must contain the same copyright notice that appears on the screen or printed page of such transmission.

The JSTOR Archive is a trusted digital repository providing for long-term preservation and access to leading academic journals and scholarly literature from around the world. The Archive is supported by libraries, scholarly societies, publishers, and foundations. It is an initiative of JSTOR, a not-for-profit organization with a mission to help the scholarly community take advantage of advances in technology. For more information regarding JSTOR, please contact support@jstor.org. 


\title{
STRONG COLLECTIONWISE NORMALITY AND M. E. RUDIN'S DOWKER SPACE
}

\author{
K. P. HART
}

\begin{abstract}
We investigate the relationship between strong collectionwise normality and some other separation properties. The conclusion is that in general there is none. In addition some properties of M. E. Rudin's Dowker space are found.
\end{abstract}

\section{Definitions and preliminaries.}

1.1. A space $Y$ is called strongly collectionwise normal (s.c.n.) [8] iff the family of all neighbourhoods of the diagonal $\Delta Y$ in $Y \times Y$ forms a uniformity.

It is known that:

Paracompact implies s.c.n. but not conversely [5];

s.c.n. implies collectionwise normal but not conversely [2].

Furthermore note that a space $Y$ is s.c.n. iff for all open $U \supset \Delta Y$ there is an open $V \supset \Delta Y$ s.t. $V \circ V \subset U$.

1.2. Let $\kappa \geqslant 2$ be a cardinal. A space $Y$ is called almost- $\kappa$-fully normal ( $\kappa$-fully normal) [6] iff every open cover $\mathscr{U}$ of $Y$ has an open refinement $\mathcal{V}$ with the following property: given $y \in Y$ and $A \subset \operatorname{St}(y, \mathcal{V})$ with $|A| \leqslant \kappa$ there is a $U \in \mathcal{U}$ s.t. $A \subset U$ (given $\mathcal{V}^{\prime} \subset \mathcal{V}$ with $\left|\mathcal{V}^{\prime}\right| \leqslant \kappa$ and $\cap \mathcal{V}^{\prime} \neq \varnothing$ there is a $U \in \mathcal{U}$ s.t. $\left.\cup \mathcal{V}^{\prime} \subset U\right)$.

It is known [6] that

paracompact $=$ fully normal $\Rightarrow \kappa$-fully normal $\Rightarrow$ almost- $\kappa$-fully normal (among Hausdorff spaces);

if $\lambda>\kappa$ then (almost-) $\lambda$-fully normal implies (almost-) $\kappa$-fully normal;

for $\kappa \geqslant \omega, \kappa$-fully normal need not imply almost- $\kappa^{+}$-fully normal;

almost-2-fully normal is equivalent to s.c.n. [2].

1.3. A space $X$ is called monotonically normal iff for every open $U \subset X$ and $x \in U$ there is an open $U_{x} \ni x$ s.t.

$$
U_{x} \cap V_{y} \neq \varnothing \Rightarrow x \in V \text { or } y \in U \text {. }
$$

Monotone normality was introduced in [4]; the above definition is in fact a characterization from [1]. It is known [4] that

monotone normality is a hereditary property;

monotonically normal implies collectionwise normal but not conversely.

Received by the editors August 22, 1980 and, in revised form, February 7, 1981.

1980 Mathematics Subject Classification. Primary 54G20, 54D15; Secondary 54G10.

Key words and phrases. Strong collectionwise normality, monotone normality, strong normality, Dowker space, $P$-space. 
1.4. Consider the following space $Y . Y=\omega_{1} \times\left(\omega_{1}+1\right)$ with each point of $\omega_{1} \times \omega_{1}$ made isolated. Cohen [2] observed that this space, which he attributed to R. H. Bing, is collectionwise normal but not s.c.n.

Eric van Douwen [9] showed that $Y$ is monotonically normal (put $U_{(\alpha, \beta)}=$ $\{(\alpha, \beta)\}$ if $\beta<\omega_{1}$ and $U_{\left(\alpha, \omega_{1}\right)}=$ any "rectangle" $(\gamma, \alpha] \times\left(\delta, \omega_{1}\right]$ contained in $U$-see 1.3) and countably paracompact (the derived set $\omega_{1} \times\left\{\omega_{1}\right\}$ is countably compact). Hence

a monotonically normal space need not be s.c.n.;

a strongly normal (= collectionwise normal and countably paracompact) space need not be s.c.n.

Furthermore $\left(\omega_{1}+1\right) \times\left(\omega_{1}+1\right)$ is compact but not monotonically normal (since it is not hereditarily normal); consequently a space which is (almost-) $\kappa$-fully normal for all $\kappa \geqslant 2$ need not be monotonically normal.

2. M. E. Rudin's Dowker space is s.c.n. In this section we show that an s.c.n. space need not be strongly normal. An example showing this must necessarily be a Dowker space (i.e. a space which is normal but not countably paracompact). We shall show that M. E. Rudin's Dowker space [7] is s.c.n.; it is in fact almost- $k$-fully normal for all finite $k$.

2.1. Description of the Dowker space $X$. We shall use the same notation as in [7]. We put

$$
\begin{aligned}
F & =\left\{f: \mathbf{N} \rightarrow \omega_{\omega} \mid \forall n: f(n) \leqslant \omega_{n}\right\}=\prod_{n=1}^{\infty}\left(\omega_{n}+1\right), \\
X & =\left\{f \in F \mid \exists i \in \mathbf{N}: \forall n \omega_{0}<\operatorname{cf}(f(n))<\omega_{i}\right\}, \\
X^{\prime} & =\left\{f \in F \mid \forall n: \omega_{0}<\operatorname{cf}(f(n))\right\} .
\end{aligned}
$$

Furthermore we define for $f, g \in F$

$$
f<g \text { iff } \forall n: f(n)<g(n), \quad f \leqslant g \text { iff } \forall n: f(n) \leqslant g(n) .
$$

We topologize $X^{\prime}$ and hence $X$ using the sets of the form $U_{f, g}=\left\{h \in X^{\prime} \mid f<h\right.$ $\leqslant g$, where $f<g$ and $f$ and $g$ run through $F$, as a base for the open sets.

It is shown in [7] that

$X$ is not countably paracompact;

$X$ is collectionwise normal;

$X^{\prime}$ is ultraparacompact (= paracompact and strongly zero-dimensional);

$X$ is $C$-embedded in $X^{\prime}$, so $X^{\prime}=v X$, the Hewitt-realcompactification of $X$.

2.2. $X$ is s.c.n. It is easy to check the following equality:

$$
U_{f, g}=X^{\prime} \cap \prod_{i=1}^{\infty}(f(i), g(i)] .
$$

From this it follows that $X^{\prime}$ (and hence $X$ ) is a subspace of $F$, if we consider $F$ to be the box product of the ordinal spaces $\omega_{1}+1, \omega_{2}+1, \ldots$ Indeed, basic open sets of $F$ intersect in basic open sets of $X^{\prime}$ and all basic open sets of $X^{\prime}$ are obtained in this way. 
Define $\varphi: F \times F \rightarrow F$ as follows:

$$
\begin{array}{cc}
\varphi(f, g)(2 i-1)= & f(i), \\
\varphi(f, g)(2 i)=g(i) & \text { for all } i .
\end{array}
$$

(i) $\varphi[F \times F]=\prod_{i=1}^{\infty}\left[0, \kappa_{i}\right]$ where $\forall i: \kappa_{2 i-1}=\kappa_{2 i}=\omega_{i}$.

$\subset: \varphi(f, g)(2 i-1)=f(i) \leqslant \omega_{i}=\kappa_{2 i-1}, \varphi(f, g)(2 i)=g(i) \leqslant \omega_{i}=\kappa_{2 i}$ for all $i, f$ and $g$.

$\supset:$ Take $f$ in the product. Define $f_{1}$ and $f_{2}$ by $f_{1}(i)=f(2 i-1), f_{2}(i)=f(2 i) \forall i$. Then

$$
f_{1}(i) \leqslant \kappa_{2 i-1}=\omega_{i} \quad \forall i, \quad f_{2}(i) \leqslant \kappa_{2 i}=\omega_{i} \quad \forall i,
$$

so $\left(f_{1}, f_{2}\right) \in F \times F$ and obviously $\varphi\left(f_{1}, f_{2}\right)=f$.

Consequently $\varphi[F \times F]$ is clopen in $F$.

(ii) $\varphi$ is obviously injective.

(iii) $\varphi\left[X^{\prime} \times X^{\prime}\right]=X^{\prime} \cap \varphi[F \times F]$.

$\subset$ : If $\omega_{0}<\operatorname{cf}(f(n)), \operatorname{cf}(g(n)), \forall n$, then certainly $\omega_{0}<\operatorname{cf}(\varphi(f, g)(n)), \forall n$.

$\supset$ : If $f \in X^{\prime} \cap \varphi[F \times F]$ then obviously $f_{1}, f_{2} \in X^{\prime}$ so $f \in \varphi\left[X^{\prime} \times X^{\prime}\right]$.

(iv) $\varphi[X \times X]=X \cap \varphi[F \times F]$.

$\subset:$ If $\forall n \omega_{0}<\operatorname{cf}(f(n))<\omega_{i}$ and $\omega_{0}<\operatorname{cf}(g(n))<\omega_{j}$ then $\forall n \omega_{0}<$ $\operatorname{cf}(\varphi(f, g)(n))<\omega_{i+j}$.

$\supset$ : If $\forall n \omega_{0}<\operatorname{cf}(f(n))<\omega_{i}$ then the same holds for $f_{1}$ and $f_{2}$.

(v) The restriction $\varphi \mid\left(X^{\prime} \times X^{\prime}\right)$, which we also denote by $\varphi$, is continuous, for obviously

$$
\varphi \leftarrow\left[U_{f, g}\right]=U_{f_{1}, g_{1}} \times U_{f_{2}, g_{2}} \quad \forall f, g
$$

(vi) $\varphi$ is also open since

$$
\varphi\left[U_{f, g} \times U_{h, k}\right]=U_{\varphi(f, h), \varphi(g, k)} \quad \forall f, g, h, k .
$$

From (i)-(vi) we see that

$\varphi[X \times X]$ and $\varphi\left[X^{\prime} \times X^{\prime}\right]$ are homeomorphic to $X \times X$ and $X^{\prime} \times X^{\prime}$ respectively;

$\varphi[X \times X]$ and $\varphi\left[X^{\prime} \times X^{\prime}\right]$ are clopen subspaces of $X$ and $X^{\prime}$ respectively.

(vii) From the above we can now conclude that $X \times X$ is normal and $C$ embedded in $X^{\prime} \times X^{\prime}$.

(viii) $X$ is s.c.n.

Let $U \supset \Delta X$ be open; by (vii), $\overline{(X \times X) \backslash U}$ and $\overline{\Delta X}=\Delta X^{\prime}$ (closures in $X^{\prime} \times$ $\left.X^{\prime}\right)$ are disjoint. So $U^{\prime}=\left(X^{\prime} \times X^{\prime}\right) \backslash \overline{((X \times X) \backslash U)}$ is an open set containing $\Delta X^{\prime}$. Since $X^{\prime}$ is ultraparacompact we can find an open $V^{\prime} \supset \Delta X^{\prime}$ such that

$$
V^{\prime}=V^{\prime} \circ V^{\prime}=\left(V^{\prime}\right)^{-1} \subset U \text {. }
$$

Now put $V=(X \times X) \cap V^{\prime}$; then we have $\Delta X \subset V=V \circ V \subset U$. 
3. Additional properties of $X$ and $X^{\prime}$. We shall exhibit some more properties of $X$ and $X^{\prime}$. We start with a lemma.

3.1. Lemma (Generalizing the Schroeder-Bernstein TheOrem). Let $Y$ be $a$ $P$-space (i.e. $G_{\delta}$-sets in $Y$ are open) and suppose $i: Y \rightarrow Z$ and $j: Z \rightarrow Y$ are embeddings such that $i[Y]$ and $j[Z]$ are clopen in $Z$ and $Y$ respectively. Then $Y$ and $Z$ are homeomorphic. Moreover, if $Y^{\prime} \subset Y$ and $Z^{\prime} \subset Z$ satisfy $i\left[Y^{\prime}\right]=i[Y] \cap Z^{\prime}$ and $j\left[Z^{\prime}\right]=j[Z] \cap Y^{\prime}$, then the homeomorphism can be chosen to map $Y^{\prime}$ onto $Z^{\prime}$.

Proof. Any standard proof of the S.-B. Theorem will do. For example: put $\mathcal{C}=\{C \subset Y \mid C$ is clopen $\}$ and define $H: \bigodot \rightarrow \bigodot$ by $H(C)=Y \backslash j[Z \backslash i[C]]$. Let $Y_{0}=Y$ and $Y_{n+1}=H\left(Y_{n}\right)\left(n \in \omega_{0}\right)$, and $Y_{\omega}=\bigcap_{n \in \omega} Y_{n}$.

$Y_{\omega} \in \mathcal{C}$ since $Y$ is a $P$-space.

$H\left(Y_{\omega}\right)=H\left(\cap_{n \in \omega} Y_{n}\right)=\bigcap_{n \in \omega} H\left(Y_{n}\right)=\bigcap_{n \in \omega} Y_{n+1}=Y_{\omega}$.

Define $h: Y \rightarrow Z$ by

$$
h(y)= \begin{cases}i(y) & \text { if } y \in Y_{\omega}, \\ j^{-1}(y) & \text { if } y \notin Y_{\omega} .\end{cases}
$$

It is easy to see that $h$ is a homeomorphism of $Y$ onto $Z$. Furthermore,

and

$$
h\left[Y^{\prime}\right]=i\left[Y^{\prime} \cap Y_{\omega}\right] \cup j^{\leftarrow}\left[Y^{\prime} \backslash Y_{\omega}\right] \subset i\left[Y^{\prime}\right] \cup j^{\leftarrow}\left[Y^{\prime}\right] \subset Z^{\prime}
$$

$$
\begin{aligned}
h\left[Y \backslash Y^{\prime}\right] & =i\left[Y_{\omega} \backslash Y^{\prime}\right] \cup j^{\leftarrow}\left[Y \backslash\left(Y^{\prime} \cup Y_{\omega}\right)\right] \\
& \subset i[Y] \backslash Z^{\prime} \cup j^{\leftarrow}\left[Y \backslash Y^{\prime}\right] \subset Z \backslash Z^{\prime}
\end{aligned}
$$

so $h\left[Y^{\prime}\right]=Z^{\prime}$.

3.2. COROLlaRY. $X^{\prime} \times X^{\prime}$ and $X^{\prime}$ are homeomorphic and the homeomorphism can be chosen to map $X \times X$ onto $X$.

Proof. $X^{\prime}$ and $X^{\prime} \times X^{\prime}$ are $P$-spaces.

$\varphi[X \times X]$ is clopen in $X^{\prime}$ and $\varphi[X \times X]=\varphi\left[X^{\prime} \times X^{\prime}\right] \cap X$.

Define i: $X^{\prime} \rightarrow X^{\prime} \times X^{\prime}$ by $\mathbf{i}(f)=\left(f, \omega_{1}\right)$, where $\omega_{1}$ is the point of $X^{\prime}$ having all coordinates equal to $\omega_{1}$.

Since $\omega_{1}$ is isolated in $X^{\prime}, \mathrm{i}\left[X^{\prime}\right]$ is clopen in $X^{\prime} \times X^{\prime}$. It is easy to check that $\mathrm{i}[X]=\mathrm{i}\left[X^{\prime}\right] \cap X \times X$. Application of 3.1 yields the desired homeomorphism.

3.3. Some consequences. 1 . It follows by induction that $\left(X^{\prime}\right)^{n}$ and $X^{\prime}$ are homeomorphic for all $n$ and that we can, in each case, choose the homeomorphism in such a way that it carries $X^{n}$ onto $X$.

2. From 1 and 3.2 it follows that $X^{n}$ is always $C$-embedded in $\left(X^{\prime}\right)^{n}$ and hence for all $n$ we have $v\left(X^{n}\right)=(v X)^{n}$, even though $X^{n}$ is not pseudocompact.

3. Also from 1 and 3.2 it follows that all finite powers of $X$ are s.c.n. Using a lemma due to Corson [3] we then see that $X$ is almost- $k$-fully normal for all finite $k$. But $X$ is not almost- $\omega_{0}$-fully normal, since Mansfield [6] proved that such spaces must be countably paracompact. 
3.4. REMARK. Even though $X$ and $X^{\prime}$ are homeomorphic to their own squares, neither space even contains a copy of its $\omega$ th power. This follows from the facts that $X$ and $X^{\prime}$ are both $P$-spaces and that no infinite product of nondegenerate spaces can be a $P$-space.

4. A remark and an acknowledgement.

4.1. In his book General topology, Á. Császár uses the name divisible for s.c.n. spaces which, in the light of the result of $\$ 2$, seems to be more appropriate.

4.2. The author would like to thank the referee for suggesting some improvements and in particular the short proof of Lemma 3.1.

\section{REFERENCES}

1. C. R. Borges, Four generalizations of stratifiable spaces, Proc. 3rd Prague Sympos. (1971), Academia, Prague, 1972, pp. 73-77.

2. H. J. Cohen, Sur une problème de M. Dieudonné, C.R. Acad. Sci. Paris 234 (1952), 290-292.

3. H. H. Corson, Examples relating to normality in topological spaces, Trans. Amer. Math. Soc. 99 (1961), 205-211.

4. R. W. Heath, D. J. Lutzer and P. L. Zenor, Monotonically normal spaces, Trans. Amer. Math. Soc. 178 (1973), $481-494$.

5. J. L. Kelley, General topology, Graduate Texts in Math., no. 27, Springer-Verlag, Berlin and New York, 1975.

6. M. J. Mansfield, Some generalizations of full normality, Trans. Amer. Math. Soc. 86 (1957), 489-505.

7. M. E. Rudin, $A$ normal space $X$ for which $X \times I$ is not normal, Fund. Math. 73 (1971), 179-186.

8. H. L. Shapiro and F. A. Smith, Paracompactness in uniform spaces, Topology Proc. 3 (1978), 179-197.

9. E. K. van Douwen, Simultaneous extension of continuous functions, Thesis, Free University, Amsterdam, 1975.

Subfaculteit Wiskunde, VriJe Universiteit, De BoelelaAn 1081, Amsterdam, The Netherlands 
http://www.jstor.org

\section{LINKED CITATIONS \\ - Page 1 of 1 -}

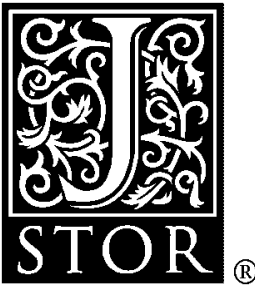

You have printed the following article:

Strong Collectionwise Normality and M. E. Rudin's Dowker Space

K. P. Hart

Proceedings of the American Mathematical Society, Vol. 83, No. 4. (Dec., 1981), pp. 802-806.

Stable URL:

http://links.jstor.org/sici?sici=0002-9939\%28198112\%2983\%3A4\%3C802\%3ASCNAME\%3E2.0.CO\%3B2-Y

This article references the following linked citations. If you are trying to access articles from an off-campus location, you may be required to first logon via your library web site to access JSTOR. Please visit your library's website or contact a librarian to learn about options for remote access to JSTOR.

\section{References}

\section{${ }^{3}$ Examples Relating to Normality in Topological Spaces}

H. H. Corson

Transactions of the American Mathematical Society, Vol. 99, No. 2. (May, 1961), pp. 205-211.

Stable URL:

http://links.jstor.org/sici?sici=0002-9947\%28196105\%2999\%3A2\%3C205\%3AERTNIT\%3E2.0.CO\%3B2-H

\footnotetext{
${ }^{4}$ Monotonically Normal Spaces

R. W. Heath; D. J. Lutzer; P. L. Zenor

Transactions of the American Mathematical Society, Vol. 178. (Apr., 1973), pp. 481-493.

Stable URL:

http://links.jstor.org/sici?sici=0002-9947\%28197304\%29178\%3C481\%3AMNS\%3E2.0.CO\%3B2-8

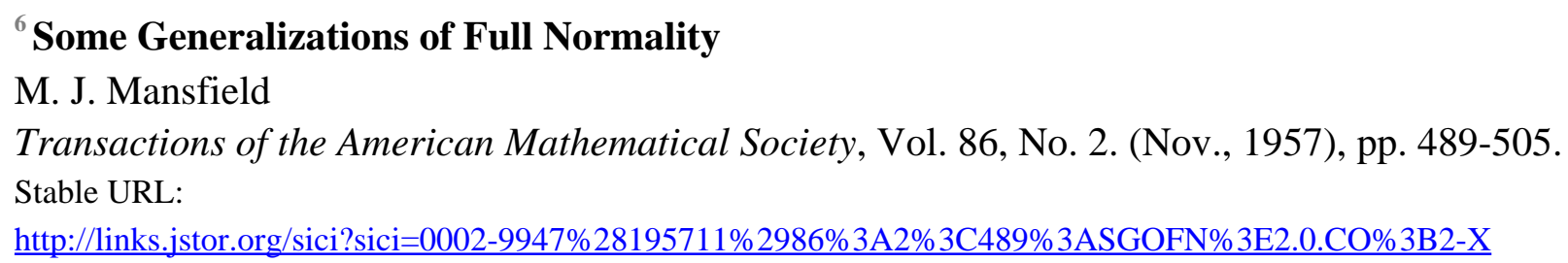

NOTE: The reference numbering from the original has been maintained in this citation list. 